\title{
Construction of Quasi Optimal Portfolio for Stochastic Models of Financial Market
}

\author{
Aleksander Janicki and Jakub Zwierz \\ Mathematical Institute, University of Wrocław, \\ pl. Grunwaldzki 2-4, 50-384 Wrocław, Poland \\ janicki@math.uni.wroc.pl \\ http://www.math.uni.wroc.pl/ ${ }^{\sim}$ janicki
}

\begin{abstract}
In the paper we propose a purely computational new method of construction of a quasi-optimal portfolio for stochastic models of a financial market. Here we present the method in the framework of a classical Black-Scholes model of a complete market (see, eg. 4], [6]), considering a well known optimal investment and consumption problem with the HARA type optimization functional. Our method is based on the idea to maximize this functional, taking into account only some subsets of possible portfolio and consumption processes. We show how to reduce the main problem to the construction of a portfolio maximizing a deterministic function of a few real valued parameters but under purely stochastic constraints. It is enough to solve several times an indicated system of stochastic differential equations (SDEs) with properly chosen parametrs.

Results of computer experiments presented here were obtained with the use of the SDE-Solver software package. This is our own professional $\mathrm{C}++$ application to Windows system, designed as a scientific computing tool based on Monte Carlo simulations and serving for numerical and statistical construction of solutions to a wide class of systems of SDEs, including a broad class of diffusions with jumps driven by non-Gaussian random measures (consult [1], 2], [5], 8, [9]).

Our method can be easily extended to stochastic models of financial market described by systems of such SDEs.
\end{abstract}

\section{Optimal Investment and Consumption Problem for Black-Scholes Model of a Financial Market}

Let us recall that an $N$ dimensional Black-Scholes model of a financial market can be described by the following system of $N+1$ SDEs

$$
\begin{aligned}
& S_{0}(t)=S_{0}(0)+\int_{0}^{t} r(s) S_{0}(s) d s, \\
& S_{n}(t)=S_{n}(0)+\int_{0}^{t} \mu_{n}(s) S_{n}(s) d s+\sum_{k=1}^{N} \int_{0}^{t} \sigma_{n, k}(s) S_{n}(s) d B_{k}(s),
\end{aligned}
$$


for $n=1, \ldots, N$ and $t \in(0, T]$, and where we have the money market with a price $S_{0}(t)$ and $N$ stocks with prices-per-share $S_{1}(t), \ldots, S_{N}(t)$, for $t \in[0, T]$.

We assume that processes $r=r(t)$, and $\mu_{n}=\mu_{n}(t)$, for $1 \leq n \leq N$, are $\mathbf{L}^{1}(\Omega \times[0, T])$-integrable, and processes $\sigma_{n, k}=\sigma_{n, k}(t)$ are $\mathbf{L}^{\overline{2}}(\Omega \times[0, T])-$ integrable on the probability space $\left(\Omega, \mathcal{F},\left\{\mathcal{F}_{t}\right\}, \mathrm{P}\right)$ with a filtration $\left\{\mathcal{F}_{t}\right\}$ generated by $N$-dimensional Brownian motion process $\left(B_{1}(t), \ldots, B_{N}(t)\right)$.

Let the stochastic processes $\eta_{0}=\eta_{0}(t)$ and $\eta_{n}=\eta_{n}(t)$, for $n=1,2, \ldots, N$, denote the number of shares of a bond and stocks, respectively. So, the value of the investor's holdings at time $t$ is represented by the wealth process, which can be represented by

$$
\mathbf{X}(t) \stackrel{\mathrm{df}}{=} \sum_{n=0}^{N} \eta_{n}(t) S_{n}(t)=\sum_{n=0}^{N} \pi_{n}(t)
$$

where

$$
\pi_{n}(t) \stackrel{\mathrm{df}}{=} \eta_{n}(t) S_{n}(t), \quad n=0,1, \ldots, N .
$$

Let $\pi(t)=\left(\pi_{1}(t), \ldots, \pi_{N}(t)\right)$. We say that the process

$$
\left(\pi_{0}, \pi\right)=\left\{\left(\pi_{0}(t), \pi(t)\right): t \in[0, T]\right\}
$$

is the portfolio process or simply portfolio of an investor.

We define the gains process $\{G(t): t \in[0, T]\}$ as a process which satisfies the following equation,

$$
d G(t)=\sum_{n=0}^{N} \eta_{n}(t) d S_{n}(t)+\sum_{n=1}^{N} S_{n}(t) \delta_{n}(t) d t
$$

where $\delta_{n}=\delta_{n}(t)$ is the so called divident rate payment process of the $n$th stock, for all $0<n \leq N$. By the consumption process we understand here any nonnegative, regular enough stochastic process $c=\{c(t): t \in[0, T]\}$. Let $x>0$ denote the initial wealth (or endowment, i.e. an amount of money an ivestor has to his disposal at time $t=0$ ), what means that we have $X(0)=x$. Let $\Gamma(t) \stackrel{\mathrm{df}}{=} x-\int_{0}^{t} c(s) d t$. We say that the portfolio $\left(\pi_{0}, \pi\right)$ is $\Gamma$-financed, when

$$
\sum_{n=0}^{N} \pi_{n}(t)=\Gamma(t)+G(t)
$$

with $G(0)=0, \quad \Gamma(0)=x$.

It is not difficult to check that if conditions (4) and (5) are satisfied, then the wealth process

$$
X \equiv X^{x, c, \pi}=\left\{X^{x, c, \pi}(t): t \in[0, T]\right\},
$$

defined by (3), can be obtained as a solution to the following Itô SDE

$$
\begin{aligned}
d X(t)=(r X(t) & -c(t)) d t+\sum_{n=1}^{N}\left(\mu_{n}(t)+\delta_{n}(t)-r\right) \pi_{n}(t) d t+ \\
& +\sum_{n=1}^{N} \pi_{n}(t) \sum_{m=1}^{N} \sigma_{n, m} B_{m}(t),
\end{aligned}
$$

with an initial condition of the form $\mathbf{X}(0)=x=\sum_{n=0}^{N} \eta_{n}(0) S_{n}(0)$. 
From (41) and (51), after application of the classical Itô formula, it follows that the following - very important in our approach - equation must be satisfied

$$
\sum_{n=0}^{N} S_{n}(t) d \eta_{n}(t)=\sum_{n=1}^{N} S_{n}(t) \delta_{n}(t) d t-c(t) d t .
$$

In optimization problems utility functions can be chosen in many different ways, however the typical choice for scientific investigations is the HARA model, what means that we chose utility function given by

$$
U^{(p)}(x) \stackrel{\text { df }}{=} x^{p} / p, \quad x>0,
$$

for $p \in(-\infty, 0)$ or $p \in(0,1)$.

The risk aversion coefficient is then defined by the formula

$$
R \stackrel{\mathrm{df}}{=}-x \frac{d^{2}}{d x^{2}} U^{(p)}(x) / \frac{d}{d x} U^{(p)}(x)=1-p .
$$

Here we are interested in the following optimization problem.

For a given utility function $U^{(p)}=U^{(p)}(c)$ and initial wealth $x>0$, we look for an optimal portfolio $\widehat{\pi}(t)=\left\{\widehat{\pi}_{1}(t), \ldots, \widehat{\pi}_{N}(t)\right\}$ and an optimal consumption process $\widehat{c}=\widehat{c}(t)$, such that for the value function of the form

$$
V_{c, \pi}(x) \stackrel{\mathrm{df}}{=} \mathrm{E}\left[\int_{0}^{T} U^{(p)}(c(t)) \mathrm{e}^{-\int_{0}^{t} \beta(s) d s} d t\right]
$$

the following condition is satisfied

$$
V_{\hat{c}, \hat{\pi}}(x)=\sup _{(c, \pi) \in \mathcal{A}(x)} V_{c, \pi}(x) .
$$

Here the condition $(c, \pi) \in \mathcal{A}(x)$ means, that the processes $c=c(t)$ and $\pi=\pi(t)$ are subject to the stochastic constraints, what means that $c=c(t)$ is positive on $[0, T]$ and the corresponding wealth process satisfing SDE (7) is such that

$$
X^{x, c, \pi}(t) \geq 0 \text { a.s. } \quad \text { for } t \in[0, T] .
$$

\section{An Example of a Quasi-optimal Portfolio}

An attempt to answer the question how to apply computational methods to solve directly and effectively optimizations problem (12) through analytical methods, e.g. making use of the Malliavin calculus (see [3], [7]) or trying to get a hedging strategy by constructing a relevant replicating portfolio (see eg. [4, 6], etc.) is not an obvious task.

So, our aim is to describe a method of computer construction of a quasioptimal portfolio solving approximate problem related to (12).

The method is based on the idea to maximize functional (11), taking into account only some subsets of possible portfolio processes derived from equations 
(7) and (8), and chosing the class of admissible positive consumption processes arbitrally, in a convenient reasonable way.

We show how to reduce the main problem to the construction of a portfolio maximizing a deterministic function of a few real valued parameters but under purely stochastic constraints.

In order to make the further exposition easier, we restrict ourselves to the classical one dimensional $(N=1)$ Black-Scholes model, which can be described in the following form

$$
\begin{aligned}
& S_{0}(t)=S_{0}(0)+r \int_{0}^{t} S_{0}(s) d s \\
& S_{1}(t)=S_{1}(0)+\mu \int_{0}^{t} S_{1}(s) d s+\sigma \int_{0}^{t} S_{1}(s) d B(s),
\end{aligned}
$$

for $t \in[0, T]$, and initial conditions such that $S_{0}(0)>0, S_{1}(0)>0$.

In the model (14)-(15) all paremeters, i.e. $S_{0}(0), S_{1}(0), r, \mu, \sigma$, are given positive real numbers. So, the processes $S_{0}, S_{1}$ can be described in the explicit closed form

$$
\begin{aligned}
& S_{0}(t)=S_{0}(0) \mathrm{e}^{r t}, \\
& S_{1}(t)=S_{1}(0) \mathrm{e}^{\left\{\left(\mu-\sigma^{2} / 2\right) t+\sigma B(t)\right\}} .
\end{aligned}
$$

The portfolio is now given by $\pi=\left(\pi_{0}, \pi_{1}\right)$, where

$$
\pi_{0}(t)=\eta_{0}(t) S_{0}(t), \quad \pi_{1}(t)=\eta_{1}(t) S_{1}(t), \quad t \in[0, T] .
$$

In the example we have chosen for computer experiments presented here we reduced the class of admissible portolio process to those which are of the following form

$$
\eta_{1}(t)=p_{1} S_{1}(t), \quad t \in[0, T] .
$$

We also restric ourselves to the class of consumption processes defined by

$$
c(t)=c_{0} S_{0}(t)+c_{1} S_{1}(t), \quad t \in[0, T],
$$

In (19) and (20) parameters $p_{1}, c_{0}, c_{1}$ are deterministic (real) variables, subject to some stochastic constraints, and which should fixed in an optimal way.

It is not difficult to notice that in such circumstances the wealth process $X(t)=X^{c_{0}, c_{1}, p_{1}}(t)$, defined by (7), solves the following Itô SDE

$$
\begin{aligned}
d X(t)= & \left(r X(t)+\left(\mu_{1}+\delta_{1}-r\right) \eta_{1}(t) S_{1}(t)-\left(c_{0} S_{0}(t)+c_{1} S_{1}(t)\right)\right) d t \\
& +\sigma_{1} \eta_{1}(t) S_{1}(t) d B(t), \quad t \in(0, T], \quad X(0)=x .
\end{aligned}
$$

Making use of the equation (8), it is also possible to check that the first component of the portfolio, i.e. the proces $\eta_{0}=\eta_{0}(t)$ solves the following SDE

$$
\begin{aligned}
d \eta_{0}(t) & =\left(\left(-\mu_{1}+\delta_{1}\right) p_{1} S_{1}^{2}(t)-\left(c_{0} S_{0}(t)+c_{1} S_{1}(t)\right)\right) / S_{0}(t) d t- \\
& -p_{1} \sigma_{1} S_{1}^{2}(t) / S_{0}(t) d B(t), t \in(0, T], \eta(0)=\left(x-p_{1} S_{1}^{2}(0)\right) / S_{0}(0) .
\end{aligned}
$$

In this way we arrive at the following problem. 
For a given utility function $U^{(p)}=U^{(p)}(c)$ and initial wealth $x>0$, we look for optimal values of parameters $\widehat{c_{0}}, \widehat{c_{1}}, \widehat{p_{1}}$, such that for the value function of the form

$$
V_{c_{0}, c_{1}, p_{1}}(x) \stackrel{\mathrm{df}}{=} \mathrm{E}\left[\int_{0}^{T} U^{(p)}\left(c_{0} S_{0}(t)+c_{1} S_{1}(t)\right) \mathrm{e}^{-\beta t} d t\right]
$$

the following condition is satisfied

$$
V_{\widehat{c_{0}}, \widehat{c_{1}} \widehat{p_{1}}}(x)=\sup _{\left(c_{0}, c_{1}, p_{1}\right) \in \mathcal{A}(x)} V_{c_{0}, c_{1}, p_{1}}(x) .
$$

Now the condition $\left(c_{0}, c_{1}, p_{1}\right) \in \mathcal{A}(x)$ means, that the consumption and wealth processes, defined by (20) and (21), are such that we have

$$
X^{c_{0}, c_{1}, p_{1}}(t) \geq 0 \text { a.s., } \quad c_{0} S_{0}(t)+c_{1} S_{1}(t) \geq 0 \text { a.s. for } t \in[0, T] .
$$

We see that, having to our disposal stochastic processes solving SDEs 14, (15), and (21), we are able to solve the problem (23)-(25). In order to get values of the value function (23) using the $S D E$-Solver software it is enough to solve the system of two equations

$$
\begin{aligned}
d Y(t) & =\left(c_{0} r S_{0}(t)+c_{1} \mu_{1} S_{1}(t)\right) d t+c_{1} \sigma_{1} S_{1}(t) d B(t), \\
d Z(t) & =U^{(p)}(Y(t)) \mathrm{e}^{\beta t} d t,
\end{aligned}
$$

for $t \in(0, T]$, and with initial conditions $Y(0)=c_{0} S_{0}(0)+c_{1} S_{1}(0), Y(0)=0$, and finally to compute

$$
V_{c_{0}, c_{1}, p_{1}}(x)=\mathrm{E} Z(T) .
$$

Then, making use of formulae (22), (19), (18), and (20), one can easily construct quasi optimal portfolio and quasi optimal consumption processes.

\section{Results of Computer Experiments}

We solved the optimization problem desribed by formulae (14)-25), with the following fixed values of constant parameters: $T=1, r=0.2, \mu_{1}=0.15, \delta_{1}=$ $0.05, \sigma_{1}=0.35, \beta=0$ and also $\beta \in\{0,0.1,0.2,0.4,0.8\}, x=50, S_{00}=50$, $S_{10}=50$.

The optimal solution for $\beta=0.0$ is of the following form:

$$
\widehat{c}_{0}=1.0, \quad \widehat{c}_{1}=-0.01, \quad \widehat{p}_{1}=0.0, \quad V_{\widehat{c}_{0}, \widehat{c}_{1}, \widehat{c p}_{1}}=14.8
$$

From a large amount of data obtained in our experiments we present here the optimal solution for $\beta=0$, including Fig. 1 presenting the convex subset of admissible parameters $\left(c_{0}, c_{1}, p_{1}\right)$ in $R^{3}$, which can be of independent interest and the Fig. 2 containing the values of the value function $V_{c_{0}, c_{1}, p_{1}}$. 


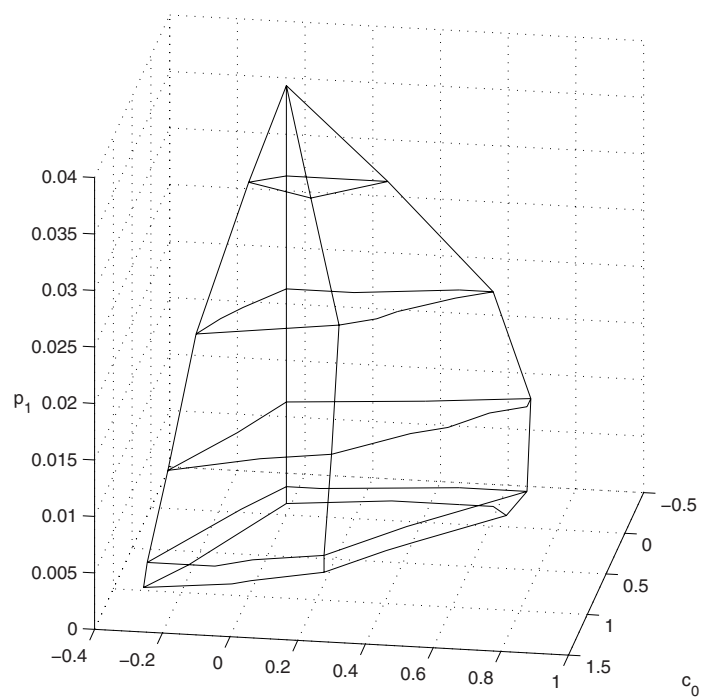

c.

Fig. 1. The convex set in $R^{3}$ containing all admissible values of parameters $V_{c_{0}, c_{1}, p_{1}}$

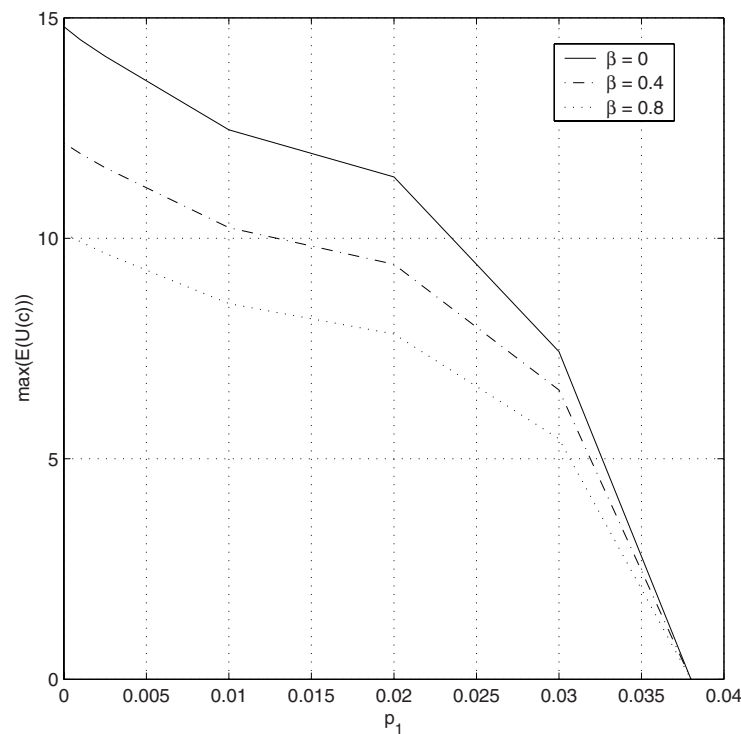

Fig. 2. Dependence of function $V_{c_{0}, c_{1}, p_{1}}$ on parameter $p_{1}$ for 3 values of $\beta$ 
In Table 1 below some values of function $V_{c_{0}, c_{1}, p_{1}}$, indicated in Fig. 2 for 3 different values of $\beta$, are presented with corresponding values of parameters $c_{0}, c_{1}, p_{1}$.

Table 1. Values of $V_{c_{0}, c_{1}, p_{1}}$ from (23)

\begin{tabular}{|c|c|c|c|c|}
\hline $\mathbf{V}_{c_{0}, c_{1}, p_{1}}(x)$ & $\mathbf{c}_{\mathbf{0}}$ & $\mathbf{c}_{\boldsymbol{1}}$ & $\mathbf{p}_{\mathbf{1}}$ & $\beta$ \\
\hline $\mathbf{1 4 . 8}$ & $\mathbf{1 . 0 0}$ & $\mathbf{- 0 . 0 1}$ & $\mathbf{0 . 0 0}$ & 0.00 \\
\hline 11.5 & 0.30 & 0.30 & 0.00 & 0.00 \\
\hline 12.5 & 0.80 & -0.10 & 0.01 & 0.00 \\
\hline 11.5 & 0.30 & 0.30 & 0.01 & 0.00 \\
\hline 11.3 & 0.30 & 0.30 & 0.02 & 0.00 \\
\hline 13.3 & 1.00 & -0.01 & 0.00 & 0.20 \\
\hline 11.7 & 0.38 & 0.38 & 0.01 & 0.20 \\
\hline 11.3 & 0.80 & -0.10 & 0.01 & 0.20 \\
\hline 10.3 & 0.30 & 0.30 & 0.02 & 0.20 \\
\hline 12.1 & 1.00 & -0.01 & 0.00 & 0.40 \\
\hline 10.6 & 0.80 & -0.05 & 0.01 & 0.40 \\
\hline 9.4 & 0.30 & 0.30 & 0.02 & 0.40 \\
\hline 10.1 & 1.00 & -0.01 & 0.00 & 0.80 \\
\hline 7.8 & 0.30 & 0.30 & 0.02 & 0.80 \\
\hline & & & & \\
\hline
\end{tabular}

In all runs of the system of equations (14), (15), (21), (26), (27) leading to computation of values of the expression in (28) with the SDE-Solver, we had 1000 trajectories of the solution, which were constructed on the grid given by 1000 subintervals of lenght 0.001 of the interval $[0,1]$. Numerical and statistical approximation methods involved are described in [1], 2], [5], 9].

Another completely solved example (simplier one, with value function depending only on two parameters) of a quasi optimal portfolio and quasi optimal consumption processes can be found in [1]. Instead of (19), (20), and (23), the following conditions describe the optimization problem:

$$
\begin{gathered}
\eta_{0}(t)=p_{0} S_{0}(t), \quad c(t)=c_{2} X(t), \\
V_{c_{2}, p_{0}}(x) \stackrel{\mathrm{df}}{=} \mathrm{E}\left[\int_{0}^{T} U^{(p)}\left(c_{2} X(t)\right) \mathrm{e}^{-\beta t} d t\right] .
\end{gathered}
$$

Graphical representations, visualizing trajectories and some statistical properties of quasi optimal processes $\eta_{0}=\eta_{0}(t), \eta_{1}=\eta_{1}(t), X=X(t)$, and $c=c(t)$, are included there. 


\section{Conclusions}

We strongly insist that even such rough approximations of the optimal investment and consumption problem as presented here are of important practical interest. One can get quite useful ideas about properties of stochastic processes solving the problem, how they depend on parameters of the stochastic model of financial market, investor preferences, etc. Of course, much more work on improvement of suggested method of construction of quasi optimal portfolio has to be done. It is also quite obvious that the method can be easily extended onto much more sofisticated stochastic models of financial market. There are various questions of mathematical nature, that should be answered in the future, e.g. on the correctness and convergence of the proposed approximate method, when more and more parameters enlargig properly the sets of admissible portfolio and consumption processes, are included.

Our computer experiments indicate to some extent the direction of further development of computational methods and computer software useful in practical solution of such complicated problems as construction of optimal strategies for investors, when stochastic model of the financial market is investigated in the framework of a system of SDEs of Itô or another, more general, type. For example, our approach can be in a very simple and natural way implemented for parallel computing systems.

\section{References}

1. Janicki, A., Izydorczyk, A.: Computer Methods in Stochastic Modeling (in Polish). Wydawnictwa Naukowo-Techniczne, Warszawa, (2001)

2. Janicki, A., Izydorczyk, A., Grạdalski, P.: Computer Simulation of Stochastic Models with SDE-Solver Software Package. Proceedings of the 3ICCS Sankt Petersburg, Lecture Notes in Computer Science 2657 (2003) 361-370

3. Janicki, A., Krajna, L.: Malliavin Calculus in Construction of Hedging Portfolios for the Heston Model of a Financial Market. Demonstratio Mathematica XXXIV (2001) 483-495

4. Karatzas I., Shreve, S.E.: Methods of Mathematical Finance. Springer-Verlag, Berlin, (1998)

5. Kloeden, P.E., Platen, E.: Numerical Solution of Stochastic Differential Equations, 3rd ed. Springer-Verlag, New York, (1998)

6. Musiela, M., Rutkowski, M.: Martingale Methods in Financial Modelling: Theory and Applications. Springer-Verlag, New York, (1997)

7. Ocone, D.L., Karatzas, I.: A generalized Clark representation formula, with application to optimal portfolios. Stochastics and Stochastics Reports 34 (1991), 187-220

8. Protter, P.: Stochastic Integration and Differential Equations - A New Approach. Springer-Verlag, New York, (1990)

9. Rachev, S. T.: Handbook of Numerical Methods in Finance. Springer-Verlag, Berlin, New York, (2004) 